\title{
Las Ciencias Sociales en América Latina: ¡Una gran herencia a vencer!
}

\author{
David Barkin \\ Universidad Autónoma Metropolitana, Unidad Xochimilco, México \\ Email: barkin@correo.xoc.uam.mx
}

\begin{abstract}
Resumen: Las nuevas ciencias sociales reconocen su deuda con las comunidades que están forjando alternativas frente a los modelos dominantes del mercado mundial, con sus paradigmas de equilibrio y de crecimiento sostenido. Para su consecución, los propios miembros de estas comunidades se están incorporando como participantes, elaborando nuevas teorías y fortaleciendo sus estrategias para forjar nuevas sociedades y proteger sus ecosistemas.
\end{abstract} sustentabilidad.

Palabras clave: Autonomía, alternativas, comunes, excedentes,

\section{Social Sciences in Latin America: A great heritage to defeat!}

\begin{abstract}
The new socialsciencesacknowledge their debt tothe communities thatare buildingalternatives tothe dominant modelsof the global market, withits paradigmsof balance andsustained growth.Toachieve them, the members ofthese communitiesare joiningas participants, developing newtheories andstrengtheningtheir strategiesto forgenew partnerships andprotect theirecosystems.
\end{abstract}

Keywords: Autonomy, alternatives, commons, surpluses, sustainability.

\section{As Ciências Sociaisna América Latina: Umagrande herançapara vencer!}

Resumo: As novasciências sociaisreconhecem a suadívida para com ascomunidadesque estão construindoalternativas para osmodelos dominantes domercadoglobal,comseus paradigmasdeequilíbrio ecrescimento sustentado.Para alcançá-los, osmembros destas comunidadesestão a aderircomo participantes, elaborando novas teoriase fortalecendo suas estratégiaspara forjarnovas sociedades eproteger os seus ecossistemas.

Palavras-chave: Autonomia,alternativas,comum;excedentes; sustentabilidade.

Hoy en día las ciencias sociales que dominan en nuestra región están condicionadas por diversas propuestas metodológicas que exigen 
cada vez más sofisticación y mayor atención a los detalles cuantitativos, proponiendo trazar el pasado, describir el presente y predecir el futuro. Su cometido es identificar las principales tendencias globales y rastrear sus impactos en las diversas dimensiones de nuestras vidas, como sociedad, como grupos sociales, y como individuos. Estas metodologías suponen que estamos encaminados hacia una cada vez más plena incorporación al mercado global, con la consecuente transformación de nuestras costumbres y nuestros patrones de consumo, en fin, nuestras formas de vida, para acoplar más cabalmente a un estilo internacionalizado de vida (y de muerte). Las instituciones internacionales refrendan estas tendencias en la academia, condicionando la formulación de políticas con sus muy poderosos incentivos crediticos y comerciales.

A pesar de estas dinámicas profundamente arraigadas en la política y la academia, siguen brotando iniciativas creativas y resistencias sociales cada vez más militantes que dan vida a las fuerzas subyacentes que insisten en forjar alternativas diversas para una sociedad más justa y en equilibrio con el planeta. Estas iniciativas han sido retomadas por una parte de los intelectuales quienes estamos construyendo nuevas epistemologías, nuevas formas de analizar el mundo, aprendiendo de los pueblos que uniéndonos con ellos en sus esfuerzos de construir diferentes formas de analizar la dinámica social y hacer viables las propuestas de estas bases sociales. La búsqueda de marcos analíticos para esta construcción no es nueva: hay una larga tradición en las ciencias sociales para generar ontologías que expliquen mejor las formas del ejercicio del poder, las formas de empobrecimiento y polarización que hoy en día están llegando a cimas jamás conocidas en la historia de la humanidad, oprimiendo a la raza humana con procesos de degradación y destrucción ambiental que pone en cuestión la posibilidad de la propia continuidad de las sociedades, tal como las conocemos (Kolbert, 2014).

Ya no es necesario remontarnos al surgimiento de los grandes debates y luchas sociales de los siglos XVIII y XIX, de donde emergió el marxismo con su riquísima herencia, poniendo en el centro de la dinámica social a la lucha de clases con su posibilidad de superar la opresión histórica que avanzó a su máxima expresión con la acumulación de capital como su propia meta. Ahora, tenemos una enorme riqueza de propuestas para repensar las ciencias sociales que construyen y elaboran sobre las propuestas de antaño, rechazando la idea de sistemas en equilibrio a favor de aprender a vivir en sistemas en conflicto, de cambio continuo, de interacciones inestables. Sería presuntuoso pretender una elaboración exhaustiva de estas propuestas, pero considerar la situación actual no podría hacer caso omiso de algunos herederos de los siglos anteriores: me refiero a pensadores contrahegemónicos que ya no están con nosotros pero quienes dejaron marcadas huellas en nuestro trabajo como R. Luxemburg (1912), V.I. Lenin (1917), M. Gandhi (1933), K. Polanyi (1944), I. Illich (1973) y P. Freire (1978). Muchos de quienes contribuyen a este número especial de Polis son también algunos otros formadores importantes de este nuevo pensamiento, con especial énfasis en las aportaciones originadas en América Latina. 
Sin embargo, nuestra aportación a ésta se concentra en otra dimensión de la transformación de las ciencias sociales: el reconocimiento de las aportes de los diversos pueblos alrededor del mundo para la comprensión de sus situaciones, y como resultado, de nuestras realidades y, quizás más importante, de nuestras potencialidades. Este reconocimiento nos ofrece la posibilidad de entender, a través de los ojos y las mentes de otros, las limitaciones de las ciencias sociales actuales y la necesidad de integrar sus "insights" (entendimientos) para que podamos sobrevivir frente a las múltiples crisis que nos achacan, las económicas, culturales, ambientales, en fin la actual crisis existencial.

Hace poco, convocamos junto con Antonio Elizalde, a un seminario sobre los "nuevos paradigmas en las ciencias sociales" que se volvió número monográfico de esta revista Polis $N^{o} 33(2012)^{1}$. Señalábamos "la imposibilidad [de los paradigmas dominantes de resolver] los problemas endémicos de la humanidad: guerra y violencia (fraternidad); pobreza e inequidad social (igualdad); y opresión (libertad)."Seguimos convencidos de que en "las prácticas ancestrales de las comunidades campesinas e indígenas, así como en sus formas actuales de organización y comportamiento, es donde se hallan algunos principios para la construcción de otras sociedades."

Desde hace tiempo hemos profundizado en nuestras colaboraciones con las comunidades para entender y complementar sus procesos para fortalecerse, construyendo lo que están viniendo a ser sociedades postcapitalistas. ¿De qué se trata? Lo importante de lo que estamos observando y nos están informando es que no son las comunidades aisladas y tradicionales de la literatura poética del "salvaje noble", de los pueblos destruidos por las conquistas, u oprimidas por los colonizadores.Más bien, son "los pueblos sin historia” (Wolf, 1982) que han resistido y siguen adelantándose con nuevas propuestas para forjar las sociedades post-capitalistas que les permitirán vivir y florecer en las márgenes de las sociedades cada vez más amenazadas por grupos externos o debilitadas desde adentro por sus divisiones sociales y su polarización económica.

En América Latina hay decenas de millones de personas viviendo en miles de comunidades, crecientemente entrelazadas entre sí, para defenderse y perfeccionar sus estrategias para consolidar sus capacidades de lograr un estilo de vida adecuado para 'satisfacer' sus necesidades y para seguir con sus responsabilidades para asegurar un equilibrio en sus ecosistemas. Asimismo, hay más de cien millones de campesinos agrupados en casi cien países en la organización social más grande del mundo "La Vía Campesina” que están adelantando sus propias estrategias para enfrentar la crisis alimentaria (la producción comunitaria y familiar de pequeña escala) (Martínez Torres y Rosset, 2010), mal diagnosticada por las instituciones internacionales (e.g., Rosegrant, et al., 2001) y los "mercaderes del grano" (Morgan, 1979) como un problema de la incapacidad de la agroindustria y la agricultura comercial de 'alimentar al mundo'. 
Las ciencias sociales que estamos perfeccionando en nuestros centros de trabajo y durante nuestra interacción con estos grupos protagonistas de nuevas sociedades tienen su origen en diversas cosmovisiones, múltiples maneras de visualizar las formas en que las sociedades se relacionan con sus entornos. Quizás las innovaciones más importantes de esta primera etapa en el desarrollo de nuestro trabajo han sido: a) la integración de miembros de las comunidades como parte de nuestros equipos de investigación, así como investigadores en derecho propio (Smith, 2012); y b) la convicciónde que estos trabajos no son ejercicios 'objetivos' (libre de valores) sino informados por y organizados para que el conocimiento promueva la transformación social, el fortalecimiento de sociedades con dinámicas que contrarrestan las tendencias excluyentes y polarizantes que caracterizan a las sociedades en las cuales están insertas; y c) la integración explícita de la relación entre la sociedad y los ecosistemas con su reconocimiento de lo limitado que son los recursos naturales y las posibilidades de aprovecharlos sin amenazar los equilibrios naturales planetarios (para una reflexión más detallada sobre eso, véase a Barkin, et al., 2012).

Nuestro punto de partida es la injusticia prevaleciente entre los grupos dominantes y los demás sectores, en todas las dimensiones de la vida y del conocimiento. Estas injusticias dominan a todo; las ciencias sociales las examinan para tratar de explicarlas, describirlas, sin encontrar formas para aminorarlas, mucho menos para proponer revertirlas. Sus repercusiones incluyen el desastre ambiental que amenaza la integridad misma del planeta. Los nuevos enfoques plantean otra salida, una construida por y con "los de abajo", las personas comunes, los que han sido excluidos y menospreciados, los miles de millones quienes siguen resistiendo, no sólo con acciones destructivas, sino, ahora más que nunca, con respuestas positivas que están contribuyendo a reorganizar y fortalecer sus comunidades, implantando soluciones para asegurar la producción de sus necesidades básicas, elevar la calidad de vida y conservar sus ecosistemas. De hecho, es eso lo que se trata cuando Foucault (citado por Esteva en el número de Polis mencionado arriba) decía que habría que darse cuenta de "saberes que estaban descalificados como saberes no conceptuales, como saberes insuficientemente elaborados: saberes ingenuos, saberes jerárquicamente inferiores, saberes por debajo del nivel del conocimiento o de la cientificidad exigidos” (Foucault 2002: 21).

\section{Los comunes: Una visión colectiva para superar las epistemologías dominantes}

Frente a la visión del individuo en el centro de la preocupación de las ciencias sociales, nuestro trabajo coloca a la responsabilidad para los comunes naturales y sociales como la prioridad. Los comunes trascienden con mucho la extraordinaria herencia natural que hemos recibido para abarcar la milenaria historia de la organización social que los enfoques dominantes pretenden desoír. La tradición de su gobernanza colectiva no se limita a 
los recursos naturales, tal como se ha puesto de moda con los renovados esfuerzos de protegerlos del efecto destructor de su manejo utilizando incentivos financieros para impulsar su conservación (Ostrom, 1990); aun cuando es un avance importante sobre la gestión mediante los incentivos mercantiles a propietarios individuales, su importancia no puede delimitarse simplemente con su transformación en propietarios grupales, que mantienen la idea de propiedad privada, una de las instituciones más perniciosas para el logro de la justicia.

Estas comunidades están colocando la política y la ética en el centro de la vida social -desplazando de la misma a la economía- explícitamente rechazando la supremacía de un cálculo económico para tomar decisiones fundamentales sobre su sociedad, economía, o la gestión del ecosistema.Están construyendo activamente alianzas entre ellas mismas, sin importar si están situadas en regiones contiguas o asociadas a través de organizaciones sectoriales o culturales que ofrecen plataformas para consolidar su habilidad para negociar con autoridades locales y nacionales, o de resistir la imposición de políticas o proyectos a los cuales se oponen. En el proceso, están alejándose de la hegemonía de estas epistemologías y fuerzas internacionales, forjando instituciones propias para crear espacios de mayor autonomía en las esferas políticas, sociales y productivas, defendiendo su forma de vida y sus territorios de ser absorbidos por la economía internacional o de su absoluta incautación/apropiación por el capital internacional.

Proteger, defender y gobernar los comunes son procesos complejos y riesgosos. Complejos, porque abarcan todos los aspectos de la existencia, la herencia del pasado, y las estructuras sociales y biológicas que han resultado; arriesgados, porque implica desafiar a los poderes fácticos y cuestionar la legitimidad de su “estado de derecho"; es decir, el sistema legal que ha creado y perpetuado una sociedad profundamente injusta, exacerbando las desigualdades sociales y acelerando la destrucción del medio ambiente. Esta disputa sobre la naturaleza del Estado proviene de un rechazo de los fundamentos filosóficos de orden hegemónico, basado en la idea de un sólo "contrato social” que presupone la posibilidad de aplicar normas universales, como "justicia social”, "igualdad” o incluso "democracia”, imparcialmente para atender las necesidades de todos los grupos sociales; la diversidad de los comunes así como la diversidad de los pueblos que los gestionan presuponen una multiplicidad de contratos sociales definidos en el seno de cada sociedad (Villoro, 2003). Por esta razón, también implica un rechazo prima facie de la legitimidad de las “autoridades” nacionales, que asumen su derecho a transferir recursos de la comunidad -los comunes- a terceros por cualquier razón, sin tener en cuenta el bienestar de los pueblos, las decisiones locales o consideraciones históricas y ambientales, como es la práctica común en el contexto minero, forestal y de gestión del agua, a pesar de que actualmente se extiende a cuestiones complejas de bio- y nanotecnología en muchas naciones. Por lo tanto, los esfuerzos para promover la solidaridad entre los diversos grupos sociales exigen un enfoque 
político que requiere que cada uno se libere de las instituciones sociales y políticas dominantes que son incapaces de atender sus necesidades particulares.

La incorporación de todos los miembros de la sociedad a la administración de los comunes, naturales, productivos, culturales, en fin, todo, requiere de diferentes formas de gobernanza, definidas por sus variadas herencias. Esta riqueza de experiencias, con sus distintas maneras de conocer, tan despreciada por las metodologías hegemónicas, encapsula el tesoro de sabidurías, para enfrentar e identificar soluciones a los problemas que enfrentan los pueblos, y es lo que lleva a formular "las epistemologías del sur” una nueva área de exploración que está enriqueciendo y redefiniendo las ciencias sociales en el "Sur Global” actualmente (Santos, 2009).

\section{Generando las bases para el bienestar y el equilibrio ambiental}

En las ciencias sociales hegemónicas la base del progreso social según su modelo de la dinámica social es la generación de ganancias por las empresas productivas de propiedad particular (o grupal) para ser canalizadas hacia la creación de nuevas empresas y empleos; cuando esa resulta inadecuada, se supone que una de las funciones principales del gobierno es canalizar parte de sus ingresos hacía proyectos infraestructurales y productivos que complementan a las actividades de la iniciativa privada. Una de las tragedias de los últimos lustros ha sido la incapacidad del modelo de cumplir con su cometido: las bajas tasas de crecimiento económico y la reducida demanda de los consumidores, sumidos cada vez más entre los rangos del consumo precario, acompañada por una menguante demanda por los consumidores internacionales, han contribuido a una incapacidad de impulsar un crecimiento que genere bienestar a escala global.

En contraste, las prácticas de otras sociedades que están fortaleciendo su determinación de separarse de esta dinámica social, han refrendado el significado de otro modelo de organización productiva y otra forma de gestionar sus recursos humanos, productivos y naturales.Para entender sus decisiones, los científicos sociales necesitamos readecuar nuestros análisis, incorporar a las nuevas formas de movilización social que son capaces de incorporar a todos sus miembros y exigir una real participación y compromiso en la consecución de sus objetivos. Aquí no se trata de decisiones individuales o de acciones despectivas que marginan a los desafortunados de la comunidad; su modelo de organización y participación es otra, remplazando el voluntarismo inherente en los sistemas dominantes con una nueva dinámica de apoyo mutuo y responsabilidad colectiva. En la práctica están generando nuevas teorías congruentes con su redefinición del mundo como uno de comunes, teorías que nos ofrecen otra manera de visualizar las posibilidades del progreso. 
En el centro de esta nueva teoría está una renovada capacidad de generar y controlar excedentes, recursos disponibles para proponer e implementar un cambio social ordenado y congruente con sus necesidades y posibilidades. A diferencia de las sociedades de las cuales son parte, este excedente es fruto del esfuerzo y de decisiones colectivos, generado por procesos no contemplados o imaginados en la teoría convencional. Paul Baran (1957) visualizó su significado cuando identificó la diferencia entre el excedente real y el potencial, señalando la imposibilidad de la sociedad capitalista de superar los obstáculos impuestos por la dinámica inherente en el mecanismo de la acumulación capitalista: la apropiación individual del excedente y su absoluta libertad de utilizarlo para sus propios designios, sin necesidad de recurrir a alguna autoridad superior para su aprobación. El resultado, tal como lo señaló Marx en su momento, es un creciente ejército de los desempleados y un empobrecimiento progresivo de enormes masas de personas a pesar del acaparamiento inigualable de riquezas.

Este excedente comunitario no es comparable con los acumulados en la sociedad capitalista. Aun cuando incluye gran parte de las ganancias de los emprendimientos productivos, su gran diferencia radica en el control colectivo que mantienen sobre él, tanto en los centros de trabajo como en las asambleas generales de los comuneros. Otra diferencia sustancial es la capacidad de aprovechar recursos intangibles para la realización de labores colectivas mediante movilizaciones para proyectos de beneficio colectivo (e.g, minga, tequio); aquí se trata de las muy comentadas brigadas comunitarias regidas por reglas locales y supervisadas por los órganos colectivos.

Centrando la atención en los procesos de producción y gestión del excedente dentro de los límites para satisfacer necesidades socialmente definidas y las posibilidades de sus ecosistemas, esta estructura de gestión colectiva de los diversos proyectos locales ha demostrado ser eficaz en la construcción de un marco de justicia ambiental que resulta ser tan difícil de lograr en las sociedades más grandes de las que forman parte. Como consecuencia, estas comunidades están organizándose para asegurar que sus miembros no tengan que sufrir pobreza extrema y desempleo que aquejan a otros segmentos de la sociedad.

\section{¿Superando las ciencias sociales?}

Esta realidad plantea enormes retos para las ciencias sociales: ¿Cómo ordenar estos diversos enfoques, las múltiples experiencias que estamos observando, que nos están relatando? Los académicos que han mostrado la independencia y la iniciativa de identificar y solidarizarse con ellos, están inmersos en una dinámica especial. En todas las divisiones de nuestras áreas de trabajo, nos encontramos tildados de heterodoxos en el mejor de los casos, de irrelevantes ode carentes de rigor, en otros.Las ontologías que están contribuyendo a forjar "nichos de sustentabilidad" en diversas partes del mundo exigen de explicaciones mucho más sensibles a las condiciones locales, el reconocimiento de que no se trata de una ciencia para la 
gente, sino de ciencias de la gente y por ella. En esta última sección ofrecemos ejemplos de cómo un manejo comunitario puede contribuir a lo que casi todos profesamos como los objetivos de nuestras labores.

Quizás dos de los más notables ejemplos en México de estas labores se refieren a la persistencia de los campesinos en seguir produciendo el maíz nativo que todavía es la base de la dieta nacional y su ampliamente reconocido éxito en lograr la conservación de sus bosques con base en organizaciones colectivas (ejidos y comunidades). Con la promulgación del Tratado de Libre Comercio de América de Norte en 1994, hubo muchos augurios de la caída en picada de la producción maicera por su falta de competitividad con los granos importados, sobretodo el transgénico de Estados Unidos.A diferencia de estas predicciones, los productores de pequeña escala han logrado aumentar sus rendimientos y la producción, aun cuando están también diversificando el abanico de actividades en que se ocupan para asegurar sus necesidades; modificaron sus patrones de producción, involucrándose directamente en la comercialización de productos de consumo a la población que aprecia la calidad de sus esfuerzos (Barkin, 2002).

En el ámbito forestal, las comunidades mexicanas libraron una batalla implacable por hacer valer sus derechos por el control sobre las tierras forestales que fueron capaces de conservar o de recuperar después de la Revolución. Ahora existe una gran variedad de planes de gestión vigentes, testimonio de las habilidades que las comunidades han adquirido al intentar conciliar presiones para asegurar la conservación, con la necesidad de crear empleos y generar ingresos. La literatura ofrece una detallada relación de esta variedad de estrategias, explorando el nexo entre estos enfoques y las cosmologías de las comunidades participantes, particularmente en bosques comunitarios, que constituyen el 71 por ciento de los bosques de la nación (i.e., Cronkleton et al., 2011; Barkin y Fuente, 2013; Stevens et al., 2014).

En México, la necesidad de otras ciencias sociales se hizo particularmente evidente con los sucesos posteriores al levantamiento zapatista en 1994 y el asombro en círculos ortodoxos al ver su capacidad de sobrevivencia y múltiples transformaciones (Muñoz, 2003). Con el incremento de la actividad y visibilidad de los pueblos indígenas en todo México se ha visto un reconocimiento gradual de sus aportaciones y su importancia, que ahora es estimada en más de 15\% de la población, a pesar de la creciente intensidad de las acciones represivas por parte del Estado y de otros actores, incluyendo corporaciones privadas con concesiones para la explotación de recursos naturales en sus territorios.

Los conflictos en torno al control y uso de los recursos hídricos es otra área donde se requiere repensar la capacidad actual de las ciencias sociales para resolver algunos de los problemas más candentes que está enfrentando la humanidad. La mancuerna con las empresas internacionales con su compromiso para la privatización de los sistemas de gestión de agua 
potable, alcantarillado y tratamiento muestra claramente las implicaciones del dominio del paradigma de equilibrio y, ahora, la capacidad del mercado para enfrentar los problemas que el propio sistema está generando. En este terreno se trata de una larga disputa sobre la naturaleza de la misma sociedad y de su capacidad como curador de estos recursos, que están revelándose cada vez más frágiles frente a los cambios climáticos. Las propuestas comunitarias para diseñar sistemas de rehabilitación y conservación de sus cuencas son notables en todas partes de las Américas, reflejando una vez más su capacidad de combinar saberes tradicionales, conocimientos heredados por la experiencia propia, y las aportaciones de técnicos y científicos para su comprensión y colaboración con los pueblos involucrados en estas iniciativas; es claro, la refundación misma de las ciencias sociales (Barkin, 2001; Boelens, 2011; Fogelberg, 2013).

En fin de cuentas, para la comprensión de la construcción y la reconstrucción de las sociedades en proceso, las ciencias sociales tendrán que aprender a aprender de los propios constructores. Es claro que muchos estamos involucrados en esta reformulación, incorporando lecciones de las múltiples cosmovisiones que están revalorándose y los enfoques sociales y políticos queplantean diferentes sociedades y sistemas productivos que ofrecerán posibilidades para una mejor vida en equilibrio con sus ecosistemas. 
Polis, Revista Latinoamericana, Volumen 14, No 41, 2015

\section{Nota}

${ }^{1}$ Disponible en http://polis.revues.org/8396 


\section{Bibliografía}

Baran, Paul A. (1957 [1959]), La Economía Política del Crecimiento. Fondo de Cultura Económica, México.

Barkin, David (2001), Innovaciones Mexicanas en el Manejo del Agua. Centro de Ecología y Desarrollo y Universidad Autónoma MetropolitanaXochimilco, México.

Barkin, David (2002), "The Reconstruction of a Modern Mexican Peasantry”. En The Journal of Peasant Studies, Vol. 30(1):73-90.

Barkin, David, Mario E. Fuente Carrasco y Daniel Tagle (2012) “La Significación de una Economía Ecológica Radical”, Revista Iberoamericana de Economía Ecológica, Vol. 19, http://www.redibec.org/IVO/REV19_01.pdf

Barkin, David y Mario E. Fuente Carrasco (2013), “Community forest management: Can the green economy contribute to environmental justice?”. En Natural Resources Forum. Vol. 37(3):200-210.

Boelens, Rutgerd. (2011) “Luchas y defensas escondidas. Pluralismo legal y cultural como una práctica de resistencia creativa en la gestión local del agua en los Andes”. EnAnuario De Estudios Americanos. Vol. 68(2):673703.

Cronkleton, Peter, David Bray, y Gabriel Medina (2011), “Community Forest Management and the Emergence of Multi-Scale Governance Institutions: Lessons for REDD+ Development from Mexico, Brazil and Bolivia”. En Forests. Vol. 2(2):451-473.

Esteva, Gustavo (2012), "Regenerar el tejido social de la esperanza”. En Polis N ${ }^{\circ} 33$.

Fogelberg, Kate (2013), “From adopt-a-project to permanent services: The evolution of Water For People's approach to rural water supply in Bolivia”. EnWater Alternatives. Vol. 6(2).

Foucault, Michel. (2002), Defender la sociedad. Fondo de Cultura Económica, México.

Freire, Paulo (1978), Pedagogia del oprimido. Siglo XXI, México.

Gandhi, Mohandes (1933), 'Hind Swaraj' or Indian Home Rule. Disponible en: http://www.mkgandhi.org/ebks/hind_swaraj.pdf

Illich, Ivan (1973 [1985]), La Convivencialidad. Joaquin Mortiz, México.

Kolbert, Elizabeth. (2014), The Sixth Extinction: An unatural history. Henry Holt \& Company, New York. 
Lenin, Vladimir Illich (1917), State and Revolution. Disponible en: http:// www.marxists.org/archive/lenin/works/1917/staterev/index.htm

Luxemburg, Rosa (1912), La Acumulación del Capital. Edicions Internacionals Sedov. Disponible en: https://kmarx.wordpress.com/2013/ 10/18/la-acumulacion-del-capital-rosa-luxemburg/

Martínez Torres, María Elena y Peter Rosset (2010), “La Vía Campesina: the birth and evolution of a transnational social movement”. En Journal of Peasant Studies. Vol. 37(1):149-175.

Morgan, Dan (1979), The Merchants of Grain. Penguin, New York.

Muñoz Ramírez, Gloria (2003), 10 y 20: El Fuego y La Palabra. Revista Rebeldía y La Jornada Libros, México.

Ostrom, Elinor (1990), Governing the Commons: The evolution of institutions for collective action. Cambridge University Press, New York.

Polanyi, Karl (1944[2003]), La Gran Transformación: La gran transformación: Los orígenes políticos y económicos de nuestro tiempo. Fondo de Cultura Económica, México.

Rosegrant, Mark W., Michael Paisner, Siet Meijer, y Julie Witcover (2001), Global Food Projections to 2020: Emerging trends and alternative futures. International Food Policy Ressarch Institute, Washington, DC.

Santos, Boaventura de Sousa (2009), Una Epistemología del Sur. La reinvención del conocimiento y la emancipación social. CLACSO-Siglo XXI, México.

Smith, Linda Tuhiwai. (1999 [2012]), Decolonizing Methodologies: Research and indigenous peoples. Zed Books. London.

Stevens, Caleb, Robert Winterbottom, Katie Reytar, y Jenny Springer, Jenny (2014), Securing Rights, Combating Climate Change: How Strengthening Community Forest Rights Mitigates Climate Change. World Resources Institute, Washington, DC.

Villoro, Luis (2003), Crecer, Saber Conocer. Siglo XXI, México.

Wolf, Eric R. (1982 [1987]), Europa y la gente sin historia. Fondo de Cultura Económica, México.

Recibido: 10.08.2015

Aceptado: 25.08.2015 